\title{
Effects of Fungicides and Spray Application Interval on Controlling Marssonina Blotch of Apple in the Loess Plateau Region of China
}

J. L. Dang, State Key Laboratory of Crop Stress Biology in Arid Areas and College of Plant Protection, Northwest A\&F University, Yangling, Shaanxi, 712100, China; M. L. Gleason, Department of Plant Pathology and Microbiology, Iowa State University, Ames 50011; and C. K. Niu, X. Liu, Y. Z. Guo, R. Zhang, and G. Y.Sun, State Key Laboratory of Crop Stress Biology in Arid Areas and College of Plant Protection, Northwest A\&F University

\begin{abstract}
Marssonina blotch, caused by the fungus Marssonina coronariae, is a serious foliar disease on apple in East Asia as well as in other moist temperate regions in Asia, Europe, and South America. Several fungicides were investigated for their toxicity to mycelial growth and conidial germination of the pathogen in vitro. Tebuconazole, kresoxim-methyl, hexaconazole, propiconazole, and a mixture of tebuconazole and benziothiazolinone sharply inhibited mycelial growth but had less effect on conidial germination. Field tests were conducted in a commercial orchard in Baishui County, Shaanxi Province, China, during 2012, 2013, 2014, and 2015 in order to develop recommendations for apple growers. Three applications of tebuconazole, hexaconazole, propiconazole, or a mixture of tebuconazole and benziothiazolinone at 20-day intervals

from early July to late August resulted in defoliation incidence of $<5 \%$. When sprays of Bordeaux mixture + tebuconazole, Bordeaux mixture + propiconazole, and Bordeaux mixture + tebuconazole and benziothiazolinone were alternated, the spray interval was extended to 25 days and defoliation incidence remained $<5 \%$. Based on historical records and our results, scouting for symptoms should begin in mid-June. We recommend commencing the spray period in early July in years with normal rainfall patterns, and spraying in mid- to late June in years with much rainfall. The findings of this study create a foundation for implementation of an efficient spray program against Marssonina leaf blotch in apple orchards in the Loess Plateau Region of China.
\end{abstract}

Marssonina blotch of apple, caused by the fungus Marssonina coronariae (Ellis \& Davis) Davis (teleomorph Diplocarpon mali Y. Harada \& Sawamura), was first reported in Japan (Parmelee 1971). It was later reported in Canada, China, Italy, India, and South Korea during the past several decades (Harada et al. 1974; Lee and Shin 2000; Li et al. 2008; Sharma 2000; Tamietti and Matta 2003). Rainfall triggers ascospore release from apothecium on the leaves in late spring to early summer and warm, rainy weather drives repeated cycles of secondary infection (Lee and Shin 2000; Sharma 2002, 2003; Sharma and Verma 2005). Severe infection of apple leaves leads to extensive defoliation, which can impair fruit size, color, and marketable yield as well as tree vigor and fruit-bearing capacity in subsequent years (Lee et al. 2008; Nie et al. 2005; Sharma and Sharma 2006). In India, the disease causes 65 to $80 \%$ defoliation prior to harvest (Sharma and Verma 2005). In China, the predominant apple-producing country in the world, Marssonina blotch is widely distributed in the major apple production regions, causing severe economic losses ( $\mathrm{Li}$ et al. 2008).

Because almost all commercial cultivars of apple are susceptible, spraying fungicides is the predominant control option (Bala et al. 2001), although orchard pruning and sanitation of fallen leaves may also reduce disease pressure (Takahashi et al. 2014). In India, a protectant program of fungicide sprays has been adopted (Kumar and Kumar 2014; Sharma and Sharma 2006). Fungicides with protective or curative activity against the pathogen include mancozeb, dodine, metiram, carbendazim, thiophanate-methyl, kresoxim-methyl, pyraclostrobin + metiram (Cabrio Top), and difenoconazole (Takahashi et al. 2014). In China, one to three applications of the demethylation-inhibiting fungicides tebuconazole, flusilazole, propiconazole, or prochloraz from July to August at 7- to 10-day intervals resulted in significant control of Marssonina blotch (Dong et al. 2009; Li et al. 2005; Li et al. 2008, 2013). Unfortunately, exclusive reliance on single-site mode-of-action fungicides for leaf blotch management has led to development of fungicide resistance (Tanaka et al. 2000) as well as the presence of unacceptable

Corresponding author: G. Y. Sun; E-mail: sgy@nwsuaf.edu.cn

Accepted for publication 18 November 2016.

() 2017 The American Phytopathological Society residues on fruit. However, alternating applications of a single-site fungicide with applications incorporating multiple sites of action may be a more promising option.

Most previous studies of Marssonina blotch in China have taken place in the Bohai Gulf Region in eastern China (Dong et al. 2009; Li et al. 2008, 2013), where epidemics typically arise in late spring to early summer. However, the disease is also a major problem in apple orchards located hundreds of kilometers further west, in the Loess Plateau Region of central and western of China. The climate in the Loess Plateau Region is drier than in the Bohai Gulf Region but summer rains trigger mid- to late-season leaf blotch epidemics in this region. Given this regional difference in epidemic risk, fungicide-spray schedules developed for the Bohai Gulf Region may need to be modified for optimal use in the Loess Plateau Region.

In recent decades, the predominant practice of protecting individual apple fruit with bags during fruit development has led growers in the Loess Plateau Region to underestimate the risk of damage due to Marssonina blotch, with the result that orchards may become almost totally defoliated before harvest. Many growers apply tebuconazole, the most widely used fungicide against leaf blotch in this region, once or twice at 10- to 15-day intervals before bagging, as well as two or three times at 30-day intervals after fruit are bagged at an early stage of fruit development. The reality that tebuconazole resistance has become widespread and that its primary substitute, Bordeaux mixture, is difficult to mix and apply adds urgency to the search for alternative management strategies.

The present research reports the results of laboratory tests to determine toxicity of widely used fungicides to mycelium and conidia of the pathogen, and field tests to determine effective fungicide-spray strategies against Marssonina blotch in the Loess Plateau Region of northwestern China. We evaluated the effects of fungicide combinations, spray intervals, and spray program initiation dates in an effort to derive rational disease-control practices for growers in the region.

\section{Materials and Methods}

Fungal pathogen. $M$. coronariae isolate H11730, collected in 2011 from a commercial orchard and stored in the Fungal Herbarium of Northwest A\&F University, Yangling, Shaanxi, was cultured on potato dextrose agar (PDA) at $25^{\circ} \mathrm{C}$ in darkness for 1 month before use for in vitro experiments to assess mycelial growth. The conidia used to assess conidial germination were 
scraped and washed from diseased leaves obtained from commercial orchards near Yangling.

Screening sensitivity to fungicides in vitro. Fungicide screening was conducted on solid media to assess conidial germination and on liquid media to assess mycelial growth. Five concentrations of each of the fungicides difenoconazole, hexaconazole, kresoximmethyl, myclobutanil, propiconazole, tebuconazole, a mixture of tebuconazole and benziothiazolinone, and Bordeaux mixture were added to $2 \%$ water agar (WA) (Tables 1 and 2). Distilled water was used as the control. Spore suspensions of 1 to $5 \times 10^{5}$ spores $/ \mathrm{ml}$ (estimated by hemacytometer) were made with sterile water and conidia from diseased leaves. The spore suspension $(10 \mu \mathrm{l})$ was pipetted onto each of three replicate plates per treatment. The inoculum was spread on the WA plates with a glass spreader. The number of germinated spores and total number of spores were counted after $12 \mathrm{~h}$ in darkness at $25^{\circ} \mathrm{C}$ and relative humidity $>90 \%$; germination inhibition percentage was calculated as [(number of germinated conidia in nonfungicide control) (number of germinated conidia in treatment)]/(number of germinated conidia in nonfungicide control) $\times 100$.

Potato and carrot dextrose agar (PCDA; $2 \mathrm{~g}$ of fresh potato, $2 \mathrm{~g}$ of fresh carrot, $2 \mathrm{~g}$ of dextrose, $1.2 \mathrm{~g}$ of agar, and $100 \mathrm{ml}$ of distilled water) was used to make mycelial culture suspensions for in vitro trials. Mycelial culture suspensions were made by scraping mycelium from $0.5-\mathrm{cm}$ diameter agar disks from PCDA cultures incubated in darkness at $25^{\circ} \mathrm{C}$ for a month and grinding it with a mortar and pestle in $1 \mathrm{ml}$ of sterile water, then diluting the mixture in $25 \mathrm{ml}$ of sterile water. Five concentrations of fungicides (Tables 1 and 2) were added into mixtures in Erlenmeyer flasks consisting of $50 \mathrm{ml}$ of potato and carrot dextrose broth liquid medium (PCD; $1 \mathrm{~g}$ of fresh potato, $1 \mathrm{~g}$ of fresh carrot, $1 \mathrm{~g}$ of dextrose, and $50 \mathrm{ml}$ of distilled water); the control was PCD without fungicide. Three replicate flasks were prepared per treatment. Each flask was inoculated by adding $1 \mathrm{ml}$ of mycelial culture (obtained by transferring acervuli from leaves and performing serial transfers on PDA to achieve pure cultures) suspension, prepared as described above. After incubation on a shaker table ( 100 revolutions $\left.\mathrm{min}^{-1}\right)$ at $25^{\circ} \mathrm{C}$ for 14 days, the mycelium was harvested by filtration through a quantitative filter paper $(110 \mathrm{~mm}$; Fushun Dongyang Industrial and Trading Co. Ltd.) in a Buchner funnel and weighed after being dried for $1 \mathrm{~h}$ in an oven at $80^{\circ} \mathrm{C}$. The mycelial growth inhibition percentage was calculated as [(mycelium dry weight of nonfungicide control) - (mycelium dry weight of treatment $)] /($ mycelium dry weight of nonfungicide control $) \times 100$.

Field location and plant material. Field studies were conducted in 2012, 2013, 2014, and 2015 in a 0.25-ha, 20-year-old block of apple trees ('Fuji', rootstock M26) in a commercial orchard near Dukang Town, Baishui County, Shaanxi Province ( $\left.35^{\circ} 18^{\prime} \mathrm{N}, 109^{\circ} 58^{\prime} \mathrm{E}\right)$. Spacing between trees was $3 \mathrm{~m}$ within rows and $4 \mathrm{~m}$ between rows (typical design in China), with nonsprayed guard rows separating treatment rows. Tree height averaged $4 \mathrm{~m}$. The experimental design was a randomized complete block; plots consisted of five contiguous trees within treatment rows and there were four replications per treatment. The plots were rerandomized in each year of the study.
Field experiments. Eight fungicides were tested to compare their impact on incidence of leaf symptoms caused by Marssonina blotch. Treatments included applying fungicides at various time intervals once, twice, or three times per season. A control treatment received no fungicide sprays.

Seven systemic fungicide treatments and four combined protectantsystemic fungicide treatments were tested in 2012, as follows. (i) Treatments with one fungicide product were tebuconazole, kresoxim-methyl, myclobutanil, hexaconazole, difenoconazole, propiconazole, and a commercial mixture of tebuconazole and benziothiazolinone. (ii) Treatments that included alternations of two fungicide products were Bordeaux mixture + tebuconazole, Bordeaux mixture + kresoxim-methyl, Bordeaux mixture + a mixture of tebuconazole and benziothiazolinone, and Bordeaux mixture + propiconazole. Bordeaux mixture alone was used as a fungicide control. All single-product systemic fungicide treatments were sprayed twice on 5 and 20 August, and disease incidence was surveyed on 15 September 2012. The corresponding application dates of the combined protectant and systemic fungicide treatments were 10 and 25 July, and disease incidence was surveyed on 25 August 2012.

Based on results of the 2012 field trials, different fungicide treatments were evaluated in 2013 and 2014 to explore an optimal strategy for the control of Marssonina blotch; in these years, each treatment received either two fungicide sprays at 30-day intervals or three fungicides sprays at intervals of either 20 or 25 days (Table 3 ).

In order to evaluate efficacy of fungicide-spray initiation threshold in 2015, two treatments involving tank mixes, one with Bordeaux mixture plus tebuconazole and one with Bordeaux mixture plus propiconazole, were applied at 20-day intervals after the first spray. In 2014 , for programs initiated at $1,3,5$, or $10 \%$ disease incidence (all $\pm 0.2 \%$ disease incidence), the first applications were $8 \mathrm{July}$, 20 July, 1 August, and 19 August, respectively. The corresponding starting dates in 2015 were 12 July, 24 July, 10 August, and 30 August. Nontreated trees were used as controls. Disease severity was surveyed on 10 September 2014 and 27 September 2015.

Table 2. Concentrations of fungicides used for in vitro testing against Marssonina coronariae

\begin{tabular}{lrrrrl}
\hline Fungicides & \multicolumn{5}{c}{ Concentration $(\mathbf{m g} / \mathbf{m l})^{\mathbf{a}}$} \\
\hline Difenoconazole & 400 & 200 & 100 & 50 & 25 \\
Hexaconazole & 600 & 300 & 150 & 75 & 37.5 \\
Kresoxim-methyl & 1,000 & 500 & 250 & 125 & 62.5 \\
Myclobutanil & 800 & 400 & 200 & 100 & 50 \\
Propiconazole & 1,000 & 500 & 250 & 125 & 62.5 \\
$\begin{array}{l}\text { Mixture of tebuconazole and } \\
\quad \text { benziothiazolinone }\end{array}$ & 200 & 100 & 50 & 25 & 12.5 \\
$\quad$ Tebuconazole & 860 & 430 & 215 & 107.5 & 53.5 \\
\hline
\end{tabular}

a All concentrations were determined according to the manufacturer's recommended concentration for each fungicide.

Table 1. Fungicides used for in vitro and in vivo testing against Marssonina coronariae and Marssonina leaf blotch, respectively

\begin{tabular}{lllll}
\hline${\text { Active ingredient }(\boldsymbol{\%})^{\mathbf{a}}}$ & \multicolumn{1}{c}{ Trade name $^{\mathbf{b}}$} & Chemical family & Form $^{\mathbf{c}}$ & Manufacturer \\
\hline Difenoconazole (10) & Shelcore & Triazole & SC & Syngenta \\
Hexaconazole (30) & Order of Merit & Triazole & SC & Sunger \\
Kresoxim-methyl (50) & Green Bay & Methoxyl acrylic ester & WG & BASF \\
Myclobutanil (40) & Allure & Triazole & WG & Sunger \\
Propiconazole (25) & Pretty & Triazole & EC & Sunger \\
Mixture of tebuconazole (12.5) and & SMT tebuconazole & Triazoles + thiazolinone & SC & Huate Western \\
benziothiazolinone (1.6) & & & SC & Bayer Crop Science \\
Tebuconazole (43) & Horlicks & Triazole & WP & Cerexagri \\
Bordeaux mixture (80) & Bouillie Bordelaise RSR & Inorganic cupric & & \\
\hline
\end{tabular}

\footnotetext{
${ }^{a}$ Number in parentheses indicates percentage of active ingredients in commercial products.

${ }^{\mathrm{b}}$ Products marketed and sold in China and used in this research.

${ }^{\mathrm{c}}$ Formulation: $\mathrm{SC}=$ suspension concentrate, $\mathrm{WG}=$ water dispersible granule, $\mathrm{EC}=$ emulsifiable concentrate, and $\mathrm{WP}=$ wettable powder.

${ }^{\mathrm{d}}$ The fungicide benziothiazolinone is produced by Huate Western, Xian, China. The chemical formula for benziothiazolinone is $\mathrm{C}_{7} \mathrm{H}_{5} \mathrm{SON}$.

${ }^{\mathrm{e}}$ Bouillie Bordelaise RSR (80\% Bordeaux mixture) containing $20 \% \mathrm{Cu}^{2+}$.
} 
In addition, field studies encompassed comparison of singlefungicide treatments and tank mixes of Bordeaux mixture plus other fungicides, differences in the date of application of the first spray of the season, and extension of the time interval between sprays $(20,25$, and 30 days; Table 3 ). All sprays above were applied to runoff with a backpack, hand-powered sprayer with a hollow cone nozzle (1.2mm-diameter spray aperture).

Disease evaluation. In evaluating disease development on leaves, five branches per tree-from the east, west, south, north, and central portions of the tree-were chosen arbitrarily. Each leaf on these branches was examined for presence of foliar symptoms, and leaf scars were noted for assessment of defoliation incidence. Assessments were made at intervals of 2 weeks (2012) or 1 month (2013, 2014 , and 2015). We also surveyed immediately before the first fungicide application to determine the disease incidence to evaluate the fungicide-spray initiation threshold in 2015.

Disease severity was estimated by both incidence of diseased leaves and defoliation percentage. Defoliation was measured by counting the number of leaf scars on each marked branch. The incidence of diseased leaves was expressed as (number of diseased leaves including defoliation/ number of investigated leaves and leaf scars $) \times 100$, whereas the defoliation percentage was expressed as (number of leaf scars per branch/ number of investigated leaves and leaf scars) $\times 100$.

Data analysis. Linear regression analysis (SAS version 9.2; SAS Institute Inc., 2008) was used to evaluate the in vitro conidial germination and mycelia growth inhibition data, and median effective concentration values for $50 \%$ inhibition of growth were calculated based on the logarithm concentration and the probability value of inhibitory rate. Means and standard deviations were obtained from analysis of four replications by Tukey's honestly significant difference (HSD) mean separation test $(P=0.05)$. Means calculated in the field experiment were compared with each other on each sampling date using analysis of variance in PROC GLM of SAS. For orchard fungicide trials, significant differences among spray-interval treatments (20, 25, or 30 days) within a rating category (incidence or defoliation), fungicide regime, and year were determined using the HSD test $(P=0.05)$ for rates of control efficacy. All subsequent reference to significant effects of factors or differences among means indicates significance at $P<0.05$, unless otherwise stated.

\section{Results}

In vitro sensitivity to fungicide. Tebuconazole, kresoxim-methyl, hexaconazole, propiconazole, and the mixture of tebuconazole and benziothiazolinone exhibited strong inhibition of mycelial growth of isolate $\mathrm{H} 11730$ but had less impact on germination of conidia. In contrast, myclobutanil, difenoconazole, and Bordeaux mixture had little impact on mycelial growth but strongly inhibited conidial germination. The mixture of tebuconazole and benziothiazolinone inhibited both conidial germination and mycelial growth (Table 4).

Disease control in field experiments. In the 2012 trials, kresoximmethyl, tebuconazole, hexaconazole, propiconazole and the mixture of tebuconazole and benziothiazolinone provided the most effective suppression of Marssonina leaf spot symptoms among the singlefungicide treatments that were sprayed every 15 days throughout the season (Fig. 1). Their control efficacy (incidence $<10 \%$ ) was significantly $(P<0.05)$ greater than for myclobutanil and difenoconazole.

The tank-mixed application of kresoxim-methyl, tebuconazole, propiconazole, and the mixture of tebuconazole and benziothiazolinone with Bordeaux mixture provided significantly greater disease control than when Bordeaux mixture was applied alone (Fig. 2). Diseased leaves and defoliation of these treatments were significantly lower than those of the no-fungicide control $(P=0.05)$.

In the 2013 trials, successive applications of Bordeaux mixture at intervals of 20 or 25 days suppressed Marssonina blotch most effectively; incidence of foliar symptoms and defoliation percentage was $<20$ and $<10 \%$, respectively. However, control was poorer in 2014, when incidence of foliar symptoms was $>50 \%$ (Fig. 3A and D).

Successive applications of tebuconazole alone provided inconsistent control of Marssonina blotch. Tebuconazole alone, applied three times at 20-, 25-, or 30-day intervals, resulted in $<20 \%$ foliar symptoms in 2013 but $>50 \%$ in 2014 trials (Fig. 3B and E).

Compared with either fungicide alone, successive applications of Bordeaux mixture + tebuconazole at intervals of 20 or 25 days reduced the incidence of foliar symptoms and defoliation percentage of Marssonina blotch noticeably more than tebuconazole alone (Fig. 3C and F). At 20-day intervals, the incidence of foliar symptoms and defoliation percentage was $<6$ and $<3 \%$, respectively, in

Table 4. Inhibitory effect of fungicides against Marssonina coronariae in vitro

\begin{tabular}{lcc}
\hline & \multicolumn{2}{c}{ Mean $\mathbf{E C}_{\mathbf{5 0}}(\boldsymbol{\mu g} / \mathbf{m l})^{\mathbf{a}}$} \\
\cline { 2 - 3 } Fungicides & Mycelial growth & Germination \\
\hline $\begin{array}{l}\text { Mixture of tebuconazole and } \\
\text { benziothiazolinone }\end{array}$ & $11.4 \pm 1.1$ & $47.3 \pm 1.7$ \\
Hexaconazole & $28.7 \pm 1.5$ & $287.0 \pm 2.5$ \\
Propiconazole & $31.2 \pm 1.5$ & $359.1 \pm 2.6$ \\
Tebuconazole & $36.1 \pm 1.6$ & $199.0 \pm 2.3$ \\
Kresoxim-methyl & $87.1 \pm 1.9$ & $391.0 \pm 2.6$ \\
Myclobutanil & $188.9 \pm 2.3$ & $219.9 \pm 2.3$ \\
Difenoconazole & $121.4 \pm 2.1$ & $17.2 \pm 1.2$ \\
Bordeaux mixture & $779.2 \pm 2.9$ & $58.5 \pm 1.8$ \\
\hline
\end{tabular}

${ }^{a}$ Effective concentration to inhibit $50 \%$ growth \pm standard deviation. Mycelia were harvested after 14 days of incubation at $25^{\circ} \mathrm{C}$ in PCD liquid medium with different fungicides, then dried and weighed. Conidium germination rate of M. coronariae isolate $\mathrm{H} 11730$ was examined after $12 \mathrm{~h}$ of incubation at $25^{\circ} \mathrm{C}$ in darkness on $2 \%$ agar plates with different fungicides. Means and standard deviations obtained from analysis of four replications by the honestly significant difference test.

${ }^{\mathrm{b}}$ Proportion of tebuconazole and benziothiazolinone in the mixture was 1:1.

Table 3. Fungicide spray treatments in the orchard in Baishui County, Shaanxi Province during 2013 and 2014a

\begin{tabular}{|c|c|c|c|}
\hline \multirow[b]{2}{*}{ Treatments $^{\mathbf{b}}$} & \multicolumn{3}{|c|}{ Sequence of fungicide applications $\mathbf{s}^{\mathrm{a}}$} \\
\hline & First spray & Second spray & Third spray \\
\hline 1 & Bordeaux mixture & Bordeaux mixture & Bordeaux mixture \\
\hline 2 & Tebuconazole & Tebuconazole & Tebuconazole \\
\hline 3 & Bordeaux mixture + tebuconazole & Bordeaux mixture + tebuconazole & Bordeaux mixture + tebuconazole \\
\hline 4 & Tebuconazole & Propiconazole & Mixture of tebuconazole and benziothiazolinone \\
\hline 5 & $\begin{array}{l}\text { Bordeaux mixture }+ \text { (mixture of tebuconazole } \\
\text { and benziothiazolinone) }\end{array}$ & Bordeaux mixture & Bordeaux mixture \\
\hline 6 & Bordeaux mixture + tebuconazole & Bordeaux mixture + propiconazole & $\begin{array}{l}\text { Bordeaux mixture }+ \text { (mixture of tebuconazole } \\
\text { and benziothiazolinone) }\end{array}$ \\
\hline
\end{tabular}

\footnotetext{
${ }^{a}$ Each fungicide applied in the field was dissolved into water as the formulation of the amount of active ingredients per liter of spray solution: Bordeaux mixture ( $1 \mathrm{~g} /$ liter), tebuconazole $(0.14 \mathrm{~g} /$ liter $)$, propiconazole $(0.13 \mathrm{~g} /$ liter $)$, and the mixture of tebuconazole and benziothiazolinone (0.18 g/liter). Fungicide solution was sprayed at 3,000 liters/ha. Tebuconazole and benziothiazolinone were premixed as the proportion of 1:1.

b All treatments were applied three times at 20- and 25-day intervals or twice at 30-day intervals. In 2013 trials, all fungicides are sprayed successively three times at 20-day intervals (25 June, 15 July, and 5 August); 25-day intervals (25 June, 20 July, and 15 August); and 30-day intervals (25 June and 25 July). In 2014 trials, all fungicides were sprayed in succession at intervals of 20 days (10 July, 30 July, and 24 August), 25 days (10 July, 5 August, and 30 August); or 30 days (10 July and 10 August). Disease development was surveyed on 25 August 2013 and 10 September 2014.
} 
2013 and $<20$ and $<3 \%$, respectively, in 2014. At 25-day intervals, the incidence of foliar symptoms and defoliation percentage was $<10$ and $<4 \%$, respectively, in 2013 and $<25$ and $<5 \%$, respectively, in 2014. However, defoliation percentage was $>10 \%$ in both years when sprays were made at a 30 -day interval.

Successive applications of tebuconazole, propiconazole, and the mixture of tebuconazole and benziothiazolinone at intervals of 20 or 25 days suppressed Marssonina blotch with intermediate effectiveness; the incidence of foliar symptoms and defoliation percentage was $<8$ and $<4 \%$, respectively, in 2013 and $<30$ and $<10 \%$, respectively, in 2014. Incidence of foliar symptoms and defoliation percentage exceeded $10 \%$ in both years when the fungicides were sprayed at a 30-day interval (Fig. 4A and D).

Successive applications of Bordeaux mixture + tebuconazole, Bordeaux mixture, and Bordeaux mixture at intervals of 20 and 25 days limited incidence of foliar symptoms and defoliation percentage to $<10$ and $<5 \%$, respectively, in 2013 and $<40$ and $<10 \%$, respectively, in 2014. Defoliation percentage was $>10 \%$ in each year when sprayed at a 30-day interval (Fig. 4B and E).

Tank-mixing tebuconazole, propiconazole, and the mixture of tebuconazole and benziothiazolinone with Bordeaux mixture was significantly $(P<0.05)$ more effective than tebuconazole, propiconazole, or the mixture of tebuconazole and benziothiazolinone alone. In 2013, successive applications of Bordeaux mixture + tebuconazole, Bordeaux mixture + propiconazole, and Bordeaux mixture + the mixture of tebuconazole and benziothiazolinone at intervals of 20,25, or 30 days suppressed Marssonina blotch more effectively than other treatments in Table 3; incidence of foliar symptoms and defoliation percentage was $<10$ and $<5 \%$, respectively. In 2014, incidence of foliar symptoms and defoliation percentage was $<20$ and $<4 \%$, respectively, at a 30-day interval (Fig. 4C and F).

Effect of fungicide-spray initiation threshold. Control efficacy was affected significantly $(P<0.05)$ by the use of disease-incidence thresholds for initiation of a fungicide spray program (Fig. 5). Bordeaux mixture + propiconazole and Bordeaux mixture + tebuconazole were sprayed three times at 20-day intervals and, when spraying was initiated at $1 \%( \pm 0.2)$ or $3 \%( \pm 0.2)$ incidence of foliar symptoms, the defoliation percentage remained $<5 \%$. Both incidence of foliar symptoms and defoliation were lower when sprays were initiated at foliar symptom incidence of $1 \%( \pm 0.2)$ and $3 \%( \pm 0.2)$ than when initiated at $5 \%$ $( \pm 0.2)$ or $10 \%( \pm 0.2)$ incidence.

\section{Discussion}

We obtained a series of effective fungicides and fungicide combinations, and constructed a practical solution for suppression of Marssonina

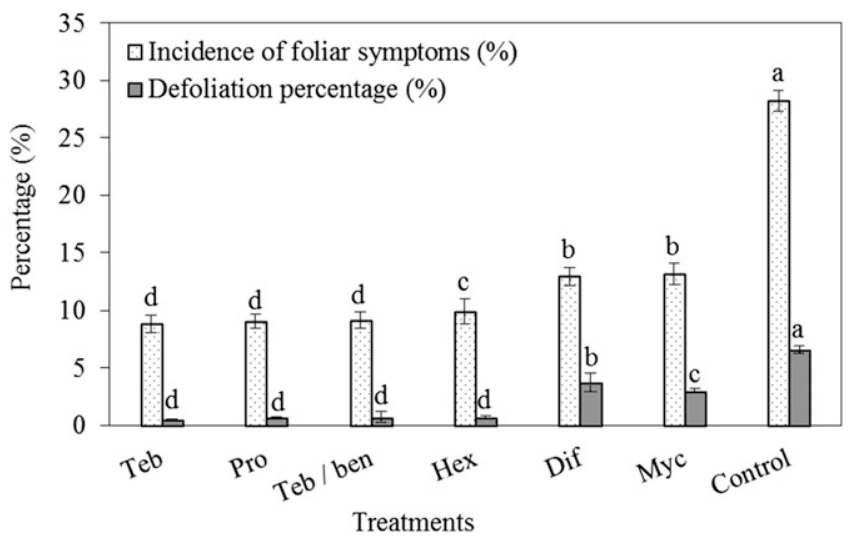

Fig. 1. Impact of seven fungicides on incidence of Marssonina blotch during 2012 in an orchard in Baishui County, Shaanxi Province. Tebuconazole (Teb), kresoxim-methyl (Krem), myclobutanil (Myc), hexaconazole (Hex), difenoconazole (Dif), the mixture of tebuconazole and benziothiazolinone (Teb/ben), and propiconazole (Pro) were sprayed twice (5 and 20 August 2012). Disease development was estimated on 15 September 2012; $n=4$. Treatments (bars of the same shading) showing different letters are significantly $(P<0.05)$ different based on Tukey's honestly significant difference mean separation test. blotch in apple orchards in the Loess Plateau Region in central and western China, which is the most important apple production region in China (Lu 1980). In vitro trials showed that tebuconazole, kresoxim-methyl, hexaconazole, propiconazole, and a mixture of tebuconazole and benziothiazolinone strongly inhibited mycelial growth but suppressed conidial germination of $M$. coronariae to a lesser extent. However, tank mixes of tebuconazole, hexaconazole, propiconazole, or the mixture of tebuconazole and benziothiazolinone with Bordeaux mixture, as well as applications of these triazole fungicides alone, resulted in effective suppression of disease development in field trials.

A fungicide spray interval of 7 to 10 days is traditional for Marssonina blotch control programs in the Bohai Gulf Region of Shandong Province (Dong et al. 2009). Under this schedule, five to six alternated sprays of Bordeaux mixture and systemic fungicides are needed after bagging to control symptoms acceptably. However, in the Loess Plateau Region, including Shaanxi Province, growers are reluctant to use traditional Bordeaux mixture, because of the inconvenience of making Bordeaux mixture. In this study, we found that both (i) successive applications of tebuconazole, propiconazole, and the mixture of tebuconazole and benziothiazolinone and (ii) the combination application of tebuconazole, propiconazole, and the mixture of tebuconazole and benziothiazolinone with Bordeaux mixture at a 20-day interval kept the defoliation percentage below $5 \%$. The incidence of foliar symptoms was $<10 \%$ in 2013 and $<35 \%$ in 2014 but the lesions on these leaves were small and expanded slowly, and the level of resulting damage was deemed commercially acceptable (J. L. Dang, unpublished data). Although we suggested an additional spray in early or mid-September for better control, Shaanxi growers reject doing this because it interferes with the start of apple harvest. Unfortunately, this reluctance can result in a high incidence of foliar symptoms and defoliation percentage.

The Loess Plateau climate is characterized by low rainfall during the early part of the apple season (spring and early summer) (Zhang et al. 2014). However, the July-to-August period is typically wetter. It is important to suppress Marssonina during the higher-risk July-toAugust period-the decisive period for epidemic development. In Shandong Province, a fungicide spray interval of 10 to 15 days is suggested for Marssonina blotch control after bagging (Dong et al. 2009). Considering the facts that $M$. coronariae possesses a relatively long incubation period of 8 to 12 days (Dong et al. 2009) and there is much less rainfall in Shaanxi than that in Shandong, there is a possibility to extend the interval between sprays in Shaanxi. We extended spray intervals from 15 days (as in 2012) to 20, 25, and 30 days in 2013 and 2014, and found that the 20-day interval resulted

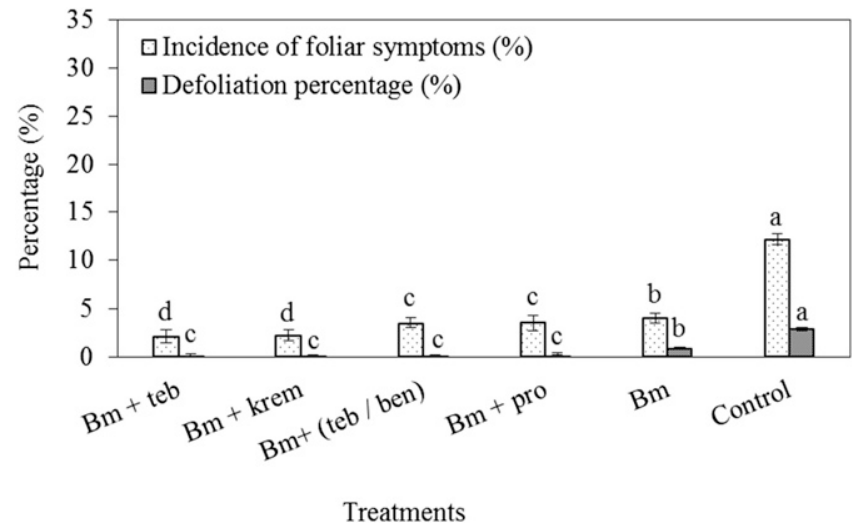

Fig. 2. Control effects of Bordeaux mixture mixed with systemic fungicides to Marssonina blotch in an orchard in Baishui County, Shaanxi Province, during 2012. Bordeaux mixture $(\mathrm{Bm})$, Bordeaux mixture + tebuconazole $(\mathrm{Bm}+\mathrm{teb})$, Bordeaux mixture + kresoxim-methyl $(\mathrm{Bm}+\mathrm{krel})$, Bordeaux mixture + the mixture of tebuconazole and benziothiazolinone $(\mathrm{Bm}+($ teb /ben)), and Bordeaux mixture + propiconazole (Bm + pro) were sprayed twice (10 and 25 July 2012). Disease development was estimated on 25 August 2012; $n=4$. Treatments (bars of the same shading) showing different letters are significantly $(P<0.05)$ different based on Tukey's honestly significant difference mean separation test. 
in $<10 \%$ incidence of symptomatic leaves and $<5 \%$ defoliation percentage for all combinations of fungicides. Spray intervals of 20 or 25 days can reduce disruption of planned spraying by rainfall, and save labor and input costs for growers. It is possible that, in a revised spray program, Shaanxi growers could adopt a 25-day spray interval for years with low to moderate weather-related risk and a 20-day interval in high-risk years-resulting in substantial savings of money and labor as well as reduced risk of resistance development. When rainfall is sufficiently sparse, it may even be possible to spray at 30day intervals, using an alternation of Bordeaux mixture + tebuconazole,
Bordeaux mixture + propiconazole, and Bordeaux mixture + the mixture of tebuconazole and benziothiazolinone.

Epidemics are closely related to temperature and relative humidity in July and August (Li et al. 2011). In Shaanxi Province, Marssonina blotch spreads rapidly in August and September, causing defoliation. To develop a warning system based on long-term forecasts, one possible approach would be use 30-, 60-, or 90-day forecasts to help plan spray programs. Another option would be development of a nearreal-time disease-warning system, using daily weather data to guide spray decisions during the season. That would require developing
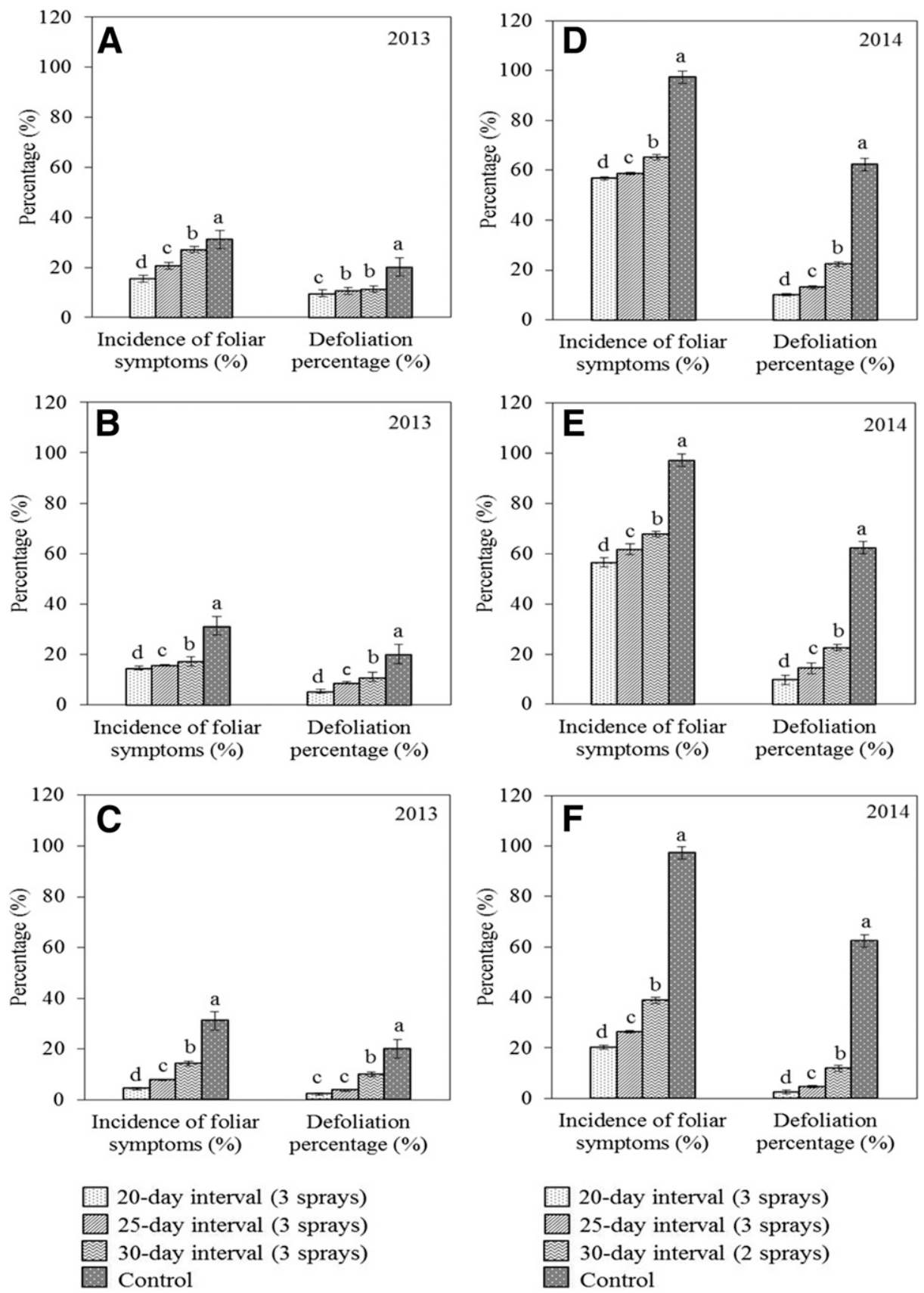

Fig. 3. Control effects of successive applications of fungicides on Marssonina blotch during 2013 and 2014 in an orchard in Baishui County, Shaanxi Province. Graphs show successive applications of $\mathbf{A}$ and $\mathbf{D}$, Bordeaux mixture alone; $\mathbf{B}$ and $\mathbf{E}$, successive applications of tebuconazole alone; and $\mathbf{C}$ and $\mathbf{F}$, successive applications of Bordeaux mixture + tebuconazole. In 2013 trials (A, B, and C), all treatments were always applied three times at 20-, 25-, and 30-day intervals after the first spray. Dates of applications were 25 June, 15 July, and 5 August for 20-day intervals; 25 June, 20 July, and 15 August for 25 -day intervals; and 25 June and 25 July for 30 -day intervals; In 2014 trials (D, E, and F), all treatments were always applied three times at 20- and 25-day intervals or twice at 30-day intervals after the first spray. Corresponding applied dates were 10 July, 30 July, and 24 August for 20-day intervals; 10 July, 5 August, and 30 August for 25-day intervals; and 10 July and 10 August for 30-day intervals. Disease development was surveyed on 25 August 2013 and 10 September 2014; $n=4$. Spray-interval treatments (20, 25 , or 30 days) within a rating category (incidence or defoliation percentage) within a fungicide regime within a year showing different letters are significantly $(P<0.05)$ different based on Tukey's honestly significant difference mean separation test. 
equations relating weather data to disease development under controlled conditions and in the field, then testing the resulting system in commercial orchards over several years, and including development of a system to relay spray recommendations to growers in a timely way.

In Shandong Province, where symptoms of Marssonina blotch begin to appear in early May, the first spray was recommended before this time (Dong et al. 2015). In Shaanxi Province, in contrast, symptoms generally do not appear until early July (Chen et al. 2012; Li et al. 2005); however, applying the first spray in May was nevertheless widely accepted by growers. Based on our trial data, the first fungicide application against Marssonina blotch was postponed to late June to early July. Therefore, our fungicide application schedule eliminated two to three sprays.

It is clear that repeated exclusive use of a fungicide class with a single-site mode of action can increase the frequency of resistant individuals in a population (Beckerman et al. 2014). Carbendazim was widely used to control Marssonina blotch in the 1980s and 1990s but lost efficacy in the 2000s owing to the possible fungicide resistance by M. coronariae (Qu et al. 2009). Triazole fungicides were
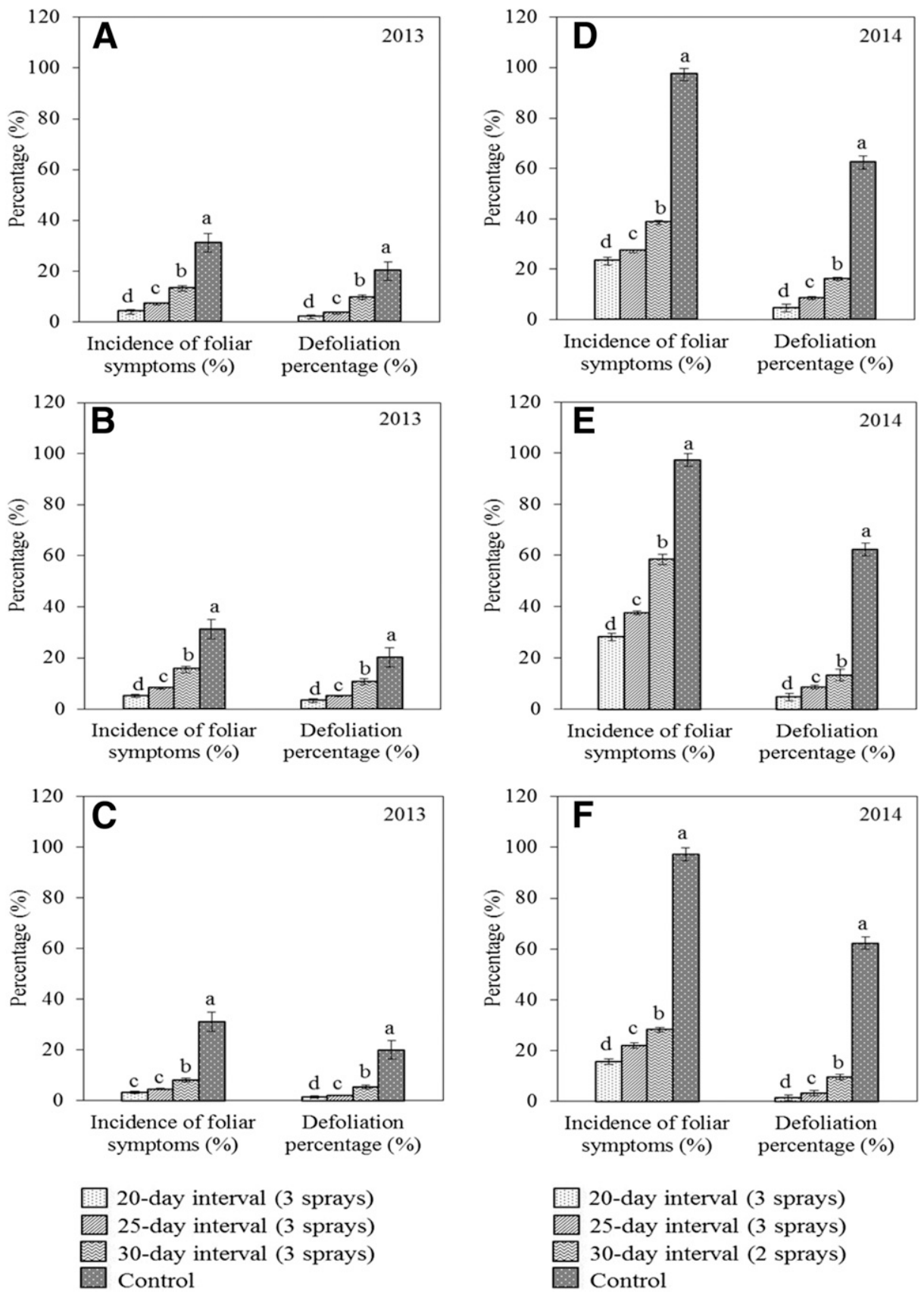

Fig. 4. Control effects of alternated applications of fungicides on Marssonina blotch during 2013 and 2014 in an orchard in Baishui County, Shaanxi Province. Graphs show alternated applications of $\mathbf{A}$ and $\mathbf{D}$, tebuconazole, propiconazole, and the mixture of tebuconazole and benziothiazolinone; $\mathbf{B}$ and $\mathbf{E}$, alternated application of Bordeaux mixture + tebuconazole, Bordeaux mixture, and Bordeaux mixture; and $\mathbf{C}$ and $\mathbf{F}$, alternated applications of Bordeaux mixture + tebuconazole, Bordeaux mixture + propiconazole, and Bordeaux mixture + the mixture of tebuconazole and benziothiazolinone. In 2013 trials (A, B, and C), all treatments were always applied three times at 20-, 25-, and 30-day intervals after the first spray. Dates of applications were 25 June, 15 July, and 5 August for 20-day intervals; 25 June, 20 July, and 15 August for 25 -day intervals; and 25 June and 25 July for 30-day intervals. In 2014 trials (D, E, and F), all treatments were always applied three times at 20- and 25-day intervals or twice at 30day intervals after the first spray. Corresponding applied dates were 8 July, 30 July, and 24 August for 20-day intervals; 8 July, 5 August, and 30 August for 25 -day intervals; and 8 July and 10 August for 30-day intervals. Disease was surveyed on 25 August 2013 and 10 September 2014; $n=4$. Spray-interval treatments (20, 25, or 30 days) within a rating category (incidence or defoliation percentage) within a fungicide regime within a year showing different letters are significantly $(P<0.05)$ different based on Tukey's honestly significant difference mean separation test. 

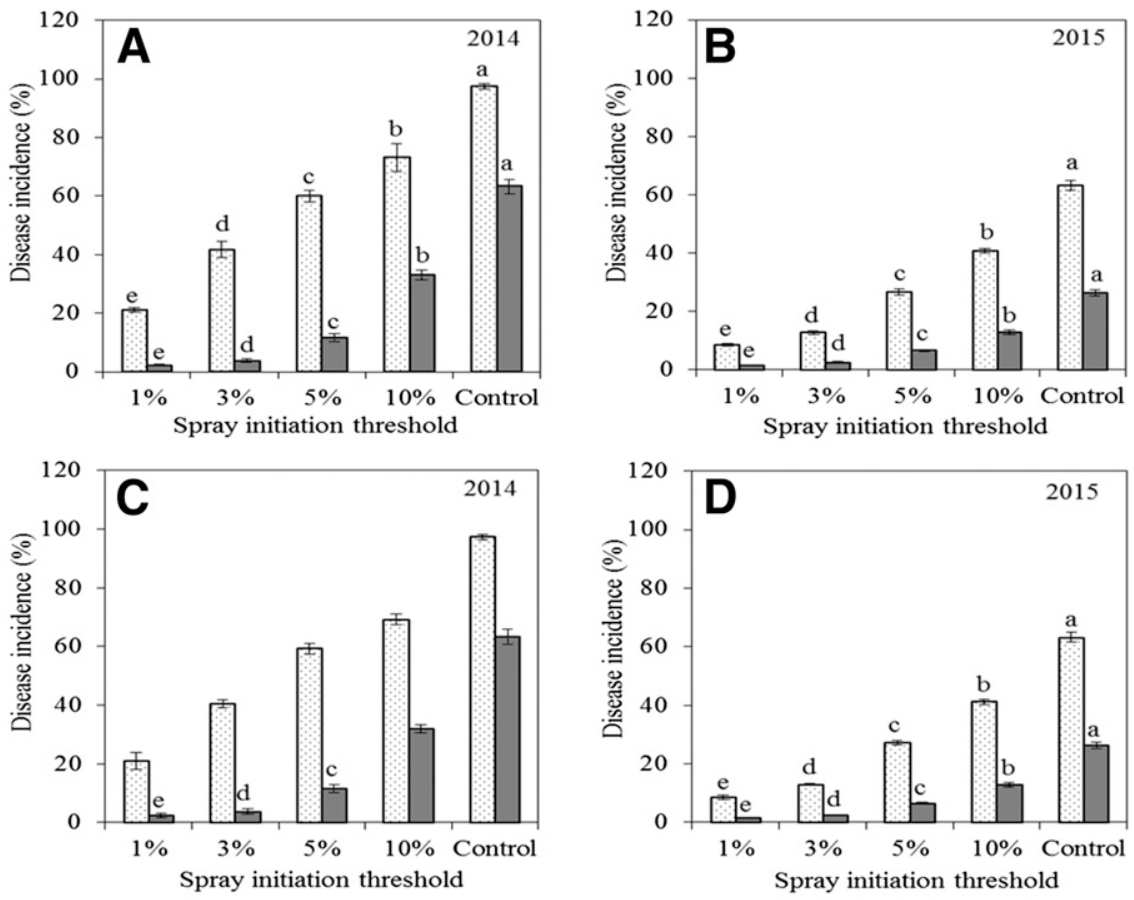

Foliar symptoms

Defoliation

Fig. 5. Effect of spray initiation threshold on control of Marssonina blotch of apple in 2014 and 2015 in an orchard in Baishui County, Shaanxi Province. Graphs show successive applications of $\mathbf{A}$ and $\mathbf{B}$, Bordeaux mixture + propiconazole or $\mathbf{C}$ and $\mathbf{D}$, Bordeaux mixture + tebuconazole for $2014(\mathrm{~A}$ and $\mathrm{C}$ ) and 2015 (B and D). Fungicides were always applied at 20-day intervals after the first spray. For programs initiated at 1, 3, 5 , or $10 \%$ disease incidence (all $\pm 0.2 \%$ disease incidence), the first applications were 8 July, 20 July, 1 August, and 19 August 2014, respectively. Corresponding starting dates in 2015 were 12 July, 24 July, 10 August, and 30 August. Nontreated trees were used as controls. Disease was surveyed on 10 September 2014 and 27 September 2015; $n=4$. Treatments (bars of the same shading) showing different letters are significantly $(P<0.05)$ different based on Tukey's honestly significant difference mean separation test.

evaluated previously for Marssonina blotch management in India (Sharma et al. 2004) and China (Dong et al. 2009; Li et al. 2008). A single triazole fungicide, tebuconazole, is commonly used to control Marssonina blotch in Shaanxi Province (Sun and Guo, unpublished data). An average of three to four fungicide sprays, primarily tebuconazole, is commonly applied after fruit bagging to suppress Marssonina blotch in Shaanxi Province orchards; however, this regime often fails to control the disease effectively (G. Y. Sun, unpublished data). In our study, three sprays of tebuconazole did not consistently suppress Marssonina blotch epidemic development, raising the possibility that $M$. coronariae in our trial orchard may possess resistance to tebuconazole, although further testing would be needed to confirm this possibility. Repeated, exclusive use of tebuconazole in the Shaanxi apple industry may have set the stage for resistance development. Based on our trails, tank-mixing Bordeaux mixture and a triazole fungicide can extend spray intervals, effectively resulting in less frequent spraying and lower dosages so as to reduce the risk of resistance development. Because both tebuconazole and propiconazole are DMI fungicides, there is a risk of developing cross resistance when using them successively. In our fungicide selection trials, kresoxim-methyl controlled Marssonina blotch more effectively than tebuconazole. We were concerned that the price of kresoxim-methyl was too high for Shaanxi growers; thus, we did not use it in combination trials with Bordeaux mixture. Nevertheless, kresoxim-methyl may be more suitable than tebuconazole for tank mixing with fungicides having other modes of action.

Alternative fungicide-spray initiation thresholds were evaluated in our field test. When the spray initiation threshold was 5 or $10 \%$ incidence of Marssonina blotch on leaves, subsequent incidence sometimes reached 60 to $70 \%$ (Fig. 5A). This can partly explain why Shaanxi growers often fail to control Marssonina blotch when they begin to spray fungicides after seeing obvious disease occurrence in orchards. When spraying was initiated at about $1 \%$ incidence of foliar symptoms, the defoliation percentage could usually be controlled to an acceptable level, except in 2014, when incidence reached about $20 \%$ (Fig. 5A).
In order to suppress Marssonina epidemics, most Shaanxi growers, lacking knowledge relating weather to disease prevention and control, begin spraying at 7 to 10-day intervals in May before bagging, then either spray for the rest of the season at 1-month intervals or initiate additional sprays when noticeable symptoms occur after bagging. Based on our findings, we recommend commencing the spray period in early July in years with normal rainfall patterns. Tank mixes of tebuconazole, the mixture of tebuconazole and benziothiazolinone, propiconazole, hexaconazole, or kresoxim-methyl with Bordeaux mixture can be applied three times at 20-day intervals. In years when rainfall is higher than normal, one protectant spray (Bordeaux mixture, mancozeb, or iprovalicarb) should be applied in mid- to late June.

Our recommendations of extended-interval and calendar-derived decision rules for determining the duration of intervals between sprays and the timing of the first spray are interim ideas to improve the efficiency and effectiveness of Marssonina blotch management. In the future, development of a warning system that explicitly relates weather conditions to disease risk should provide a substantial additional gain for growers in enhancing both reliability and costeffectiveness of their spray programs.

\section{Acknowledgments}

We thank Z. Zhao, L. Wang, Z. Du, and J. Liang in the Apple Experiment Station of Northwest A\&F University at Baishui, Shaanxi, China; Mr. Tao for providing his commercial orchard for our field experiments; and three anonymous reviewers and the Senior Editor for their constructive suggestions in revising our manuscript. This work was supported by National Natural Science Foundation of China (31371887 and 31171797), the 111 Project from Education Ministry of China (B07049), and Specialized Research Fund for the Doctoral Program of Higher Education (20130204110002) and China Agriculture Research System (CARS-28).

\section{Literature Cited}

Bala, R., Bhardwaj, L. N., Ram, V., and Sharma, G. K. 2001. Management of premature leaf falls of apple. Plant Dis. Res. 16:119-122.

Beckerman, J. L., Sundin, G. W., and Rosenberger, D. A. 2014. Do IPM concepts contribute to the development of fungicide resistance? Lessons learned from the apple scab pathosystem. Pest Manage. Sci. 71:331-342. 
Chen, P. N., Shan, H. Y., Hu, X. M., Ma, Z. Y., Feng, Z. Z., and An, D. R. 2012. Forecasting models of apple Marssonina coronaria in Northwest China. J. Biomath. 27:571-575.

Dong, X. L., Gao, Y. E., Li, B. H., Yong, D. J., Wang, C. X., Li, G. F., and Li, B. D. 2015. Epidemic dynamics of apple Marssonina leaf blotch over whole growth season in the central area of Shandong Peninsula. Sci. Agric. Sin. 48:479-487.

Dong, X. L., Luo, L., Wang, C. X., Dong, X. X., Zhang, J. L., and Li, B. H. 2009. Fungicides controlling Diplocarpon mali on apple tree and their suitable applying time. Chin. Agric. Sci. Bull. 25:190-194.

Harada, Y., Sawamura, K., and Konno, K. 1974. Diplocarpon mali sp. nov. the perfect state of apple blotch fungus Marssonina coronaria. Ann. Phytopathol. Soc. Jpn. 40:412-418.

Kumar, A., and Kumar, A. 2014. Monitored control of Marssonina blotch of apple caused by Marssonina coronaria. Indian Phytopathol. 67:70-76.

Lee, D. H., Choi, K. H., Lee, S. W., and Kim, D. A. 2008. Recent status of apple disease in the major apple producing areas in Korea. Pages 827-828 in: Report of Horticultural Research in 2008. Apple Experiment Institute, RDA Press, Wansan-gu, Jeonju-si, Korea.

Lee, H. T., and Shin, H. D. 2000. Taxonomic studies on the genus Marssonina in Korea. Mycobiology 281:39-46.

Li, B. H., Dong, X. L., Li, G. F., and Wang, C. X. 2008. The occurrence and control of Marssonina leaf blotch of apple in Yantai, Shandong Province. China Fruits 6:33-35.

Li, B. H., Wang, C. X., and Dong, X. L. 2013. Research progress in apple diseases and problems in the disease management in China. Plant Prot. 39:46-54.

Li, D. H., Zhao, Z. Y., Zhao, H. Y., Hu, X. S., Lei, Y. M., Liu, X. Y., Shang, D. D., and Gao, P. 2005. The study of regularity of apple early defoliation disease and its control by fungicide. J. Northwest Sci-Tech Univ. Agric. For. (Nat. Sci. Ed.) 33:76-80.

Li, J., Gou, L. X., Hu, X. M., Ren, F. P., Wei, J. F., and An, D. R. 2011. Effects of climate factors on the epidemic of apple Marssonina blotch in Shaanxi Province and related prediction models. Chin. J. Appl. Ecol. 22:268-272.

Lu, Q. N. 1980. The distribution and ecologic factors of apples in China. Sci. Agric. Sin. 13:46-51.

Nie, J. Y., Cong, P. H., Yang, Z. F., Li, J., and Zhang, H. J. 2005. Primary report of apple pesticide residues in China. Chin. Agric. Sci. Bull. 21:88-90.
Parmelee, J. A. 1971. Marssonina leaf spot of apple. Can. Plant Dis. Surv. 57: 91-92.

Qu, J. L., Fan, Q., Li, X. J., Zhang, Y., and Sun, H. Y. 2009. Control effect of several fungicides on apple brown spot (Marssonina mali) in field. Acta Agric. Jiangxi 21:90-91.

SAS Institute Inc. 2008. Introduction to nonparametric analysis. Pages 291-298 in: SAS/STAT 9.2 User's Guide. SAS Institute Inc., Cary, NC.

Sharma, I. M. 2003. Influence of environmental factors on the development of premature defoliation disease caused by Marssonina coronaria in apple and its management. J. Mycol. Plant Pathol. 33:89-95.

Sharma, J. N. 2000. Marssonina blotch-a new disease of apple and its control. Indian J. Plant Prot. 28:100-101.

Sharma, J. N. 2002. Managing pre-mature leaf fall in apple. Indian Hortic. July-September: 4-5.

Sharma, J. N., Sharma, A., and Sharma, P. 2004. Out-break of Marssonina blotch in warmer climates causing premature leaf fall problem of apple and its management. Acta Hortic. 662:405-409.

Sharma, J. N., and Sharma, P. 2006. Studies on Marssonina coronaria (Ell. \& J. J Davis) J. J. Davis causing Marssonina blotch of apple in Himachal Pradesh. Phytomorphology 56:61-64.

Sharma, J. N., and Verma, K. D. 2005. Integrated management of foliar and fruit disease of apple in Himachal Pradesh. J. Mycol. Plant Pathol. 35:48-52.

Takahashi, S., Sawamura, K., and Sato, Y. 2014. Marssonina blotch. Pages 46-47 in: Compendium of Apple and Pear Diseases and Pests, 2nd ed. T. B. Sutton, H. S. Aldwinckle, A. M. Agnello, and J. F. Walgenbach, eds. American Phytopathological Society, St. Paul, MN.

Tamietti, G., and Matta, A. 2003. First report of leaf blotch caused by Marssonina coronaria on apple in Italy. Plant Dis. 87:1005.

Tanaka, S., Kamegawa, N., Ito, S., and Kameya-iwaki, M. 2000. Detection of thiophanate-methyl resistant strains in Diplocarpon mali, causal fungus of apple blotch. J. Gene. Plant Pathol. 66:82-85.

Zhang, B. Q., Wu, P. T., Zhao, X. N., Gao, X. D., and Shi, Y. G. 2014. Assessing the spatial and temporal variation of the rainwater harvesting potential (19712010) on the Chinese Loess Plateau using the VIC model. Hydrol. Processes 28:534-544. 